Original Article

\title{
FORMULATION AND EVALUATION OF GLICLAZIDE NANOSPONGES
}

\author{
RAHUL S. SOLUNKE ${ }^{1}$, UDAY R. BORGE ${ }^{2}$, KRISHNA MURTHY ${ }^{3}$, MADHURI T. DESHMUKH ${ }^{4}$, RAJKUMAR V. SHETE
}

1,2,4* Department of Pharmaceutics, Rajgad Dnyanpeeth's College of Pharmacy Bhor, Pune 412206, Maharashtra, India, ${ }^{3}$ Department of Pharmacognosy, Rajgad Dnyanpeeth's College of Pharmacy Bhor, Pune 412206, Maharashtra, India, ${ }^{5}$ Department of Pharmacology, Rajgad Dnyanpeeth's College of Pharmacy Bhor, Pune 412206, Maharashtra, India Email: udayborge466@gmail.com

Received: 20 Jul 2019, Revised and Accepted: 05 Oct 2019

\section{ABSTRACT}

Objective: The objective of the present study was to develop and characterize an optimal stable nanosponges of Gliclazide (GLZ) by using the emulsion solvent diffusion method and aimed to increase its bioavailability and release the drug in sustained and controlled manner.

Methods: The GLZ nanosponge was prepared by emulsion solvent diffusion method using different drug-polymer ratios (1:1 to 1:5) Eudragit S100 is used as a polymer. Differential scanning calorimetry (DSC) and Fourier transform infrared spectroscopy (FTIR) estimated the compatibility of GLZ with polymer. All formulations evaluated for production yield, entrapment efficiency, in vitro drug release, scanning electron microscopy (SEM) and stability studies.

Results: The DSC and FTIR Studies revealed that no interaction between drug and polymer. The Production yield of all batches in the range of $73.8 \pm 0.30$ to $85.6 \pm 0.32$. Batch F3 showed the highest production yield, the entrapment efficiency of batch F3 $70.6 \pm 0.77$. The average particle size ranges from $303 \pm 2.36$ to $680 \pm 2.50 \mathrm{~nm}$. By the end of $10^{\text {th }}$ hour F3 formulation shown highest drug release was found to be $94.40 \pm 1.12 \%$. The release kinetics of the optimized formulation shows zero-order drug release. The stability study indicates no significant change in the in vitro dissolution profile of optimized formulation.

Conclusion: The results of various evaluation parameters, revealed that GLZ nanosponges would be possible alternative delivery systems to conventional formulation to improve its bioavailability, the emulsion solvent diffusion method is best method for preparation of nanosponges and release the drug in sustained and controlled manner.

Keywords: Nanosponge, Gliclazide, Controlled release, Emulsion solvent diffusion method

(C) 2019 The Authors. Published by Innovare Academic Sciences Pvt Ltd. This is an open-access article under the CC BY license (http://creativecommons.org/licenses/by/4.0/) DOI: http://dx.doi.org/10.22159/ijap.2019v11i6.35006

\section{INTRODUCTION}

Nanosponge is a modern category of material and it is made up of tiny particles with a narrow cavity of few nanometers. These narrow cavities can be filled with various types of substances these tiny particles are having capability due to which it is able to carry both hydrophilic and lipophilic drug substances [1]. Release the drug at specific target site instead of circulate through the body it will more effective for particular given dosage [2, 3]. The invention of nanosponges has become significant step towards overcoming the complexity associated with the newly developing systems. The small size and porous nature of nanosponges can bind poorly watersoluble drugs within the matrix and improve their solubility and bioavailability [4]. Nanosponges are able to entrap both hydrophilic as well as lipophilic drug molecules because of their inner hydrophobic cavities and external hydrophilic branching, thereby offering unparalleled flexibility. Nanosponge obtained by using suitable cross-linking agent also by different organic and inorganic materials [5]. The stability of these formulations over a wide range of $\mathrm{pH}$ in GI fluids and stable over $130{ }^{\circ} \mathrm{C}$ compatible with most vehicles and ingredients. Reducing dosing frequency and increase patient compliance and comfort. This drug delivery system having entrap wide variety of ingredients and reducing side effects, improve stability, increased elegance and formulation flexibility [6] The effort to improve dissolution and solubility of poorly and practically water insoluble drugs remains one of the most challenging tasks in drug development. Several methods have been introduced to increase dissolution rate and thereby oral absorption and bioavailability of such drugs. Among various approaches, Nanosponges has shown promising results in improving solubility, wettability, dissolution rate of drug and subsequently its bioavailability [7]. The nanosponges can overcome some of the shortcomings of the conventional dosage forms. GLZ is one of the second-generation sulphonylurea, antidiabetic drug which stimulates insulin release. It is used for the treatment of non-insulindependent diabetes mellitus. GLZ is classified under class II according to biopharmaceutical classification system (BCS). The drug shows low, pH dependent solubility. In acidic and neutral aqueous media, GLZ exhibits very poor solubility. This poor solubility may cause poor dissolution and unpredicted bioavailability. Preparation of nanosponges of GLZ to improve the dissolution rate of GLZ and subsequently its bioavailability. The main objective of the study was to increase the amount of dissolved drug molecules at the absorption site by increasing the dissolution rate, since for class II drugs like GLZ, in vivo dissolution rate is ratelimiting step in drug absorption. nanosponges were selected as the method of choice since it would be easier in subsequent formulating. The nanosponges were prepared at various drug-to carrier weight ratios by emulsion solvent diffusion method.

\section{MATERIALS AND METHODS}

\section{Materials}

Gliclazide (GLZ) was provided by Bal Pharma Bengaluru India. Eudragit S100 provided by Evonik Mumbai. Polyvinyl Alcohol (PVA), Dichloromethane (DCM), Triethyl citrate was obtained from chemdyes corporation Rajkot Gujrat India.

\section{Methods}

GLZ nanosponges were prepared by emulsion solvent diffusion method. In this method, two phases used are aqueous and organic. Aqueous phase consists of polyvinyl alcohol and organic phase include GLZ and Eudragit S100. After dissolving GLZ and Eudragit S100 to suitable organic solvent. This phase added slowly to the aqueous phase and stirred for two or more hours and then nanosponges are collected by filtration, washed, and then dried in air at room temperature or in vacuum oven $40^{\circ} \mathrm{C}$ for $24 \mathrm{~h}$ [8] 
Table 1: Formulation of GLZ loaded nanosponges

\begin{tabular}{lllll}
\hline Materials & F1 & F2 & F3 & F4 \\
\hline Drug: polymer(mg) & $100: 100$ & $100: 200$ & $100: 300$ & $100: 400$ \\
PVA (\%w/v) & 0.5 & 0.5 & 0.5 & 0.5 \\
Dichloromethane (ml) & 20 & 20 & 20 & 20 \\
Triethyl citrate (ml) & 0.5 & 0.5 & 0.5 & 0.5 \\
Distilled water (ml) & 100 & 100 & 100 & 20 \\
\hline
\end{tabular}

\section{Preformulation studies of pure drug}

\section{Construction of calibration curve}

Stock I.

Accurately weighed $10 \mathrm{mg}$ of GLZ was dissolved the insufficient amount of phosphate buffer 6.8 and volume was made to $10 \mathrm{ml}$ with it. (conc $1000 \mu \mathrm{g} / \mathrm{ml}$ )

Stock II

From stock-I $1 \mathrm{ml}$ sample is withdrawn by pippete and diluted to 10 $\mathrm{ml}$ of by using phosphate buffer $6.8(100 \mu \mathrm{g} / \mathrm{ml})$

Stock III

From stock II Working standard solution of strengths 5, 10, 15, 20, $25(\mu \mathrm{g} / \mathrm{ml})$ were made from the stock solution by appropriate dilution.

\section{Drug-excipient compatibility studies}

The drug and excipient compatibility studies were carried out by Fourier transform-infrared spectroscopy (FT-IR). The Potassium Bromide pellets were prepared on $\mathrm{KBr}$ press on grounding the solid powder sample with 100 times the quantity of $\mathrm{KBr}$ in mortar. The spectra recorded over the wavenumber of 4000 to $400 \mathrm{~cm}^{-1}$.

\section{Evaluation studies of prepared nanosponges}

From the results obtained by solubility and dissolution studies, nanosponges which showed better result was selected. For further characterization, nanosponges were performed to access interaction if any between the drug and polymer and also to find out what properties of polymer make them an effective material for solubility and bioavailability enhancement. In present study, the nanosponges of GLZ with Eudragit S100 were characterized by FT-IR, DSC, SEM, in vitro drug release study, production yield, entrapment efficiency etc.

\section{Production yield}

The production yield (PY) can be determined by calculating the initial weight of raw materials and final weight of nanosponges.

$$
\text { Production yield }=\frac{\text { Practical mass of nanosponges }}{\text { Theoretical mass }(\text { polymer }+ \text { drug })} \times 100
$$

The percentage yield of different batches was determined by weighing the nanosponges after drying [9].

\section{Drug entrapment efficiency}

To calculate the entrapment efficiency accurately weighed the quantity of nanosponges $(10 \mathrm{mg})$ with $5 \mathrm{ml}$ of methanol in a volumetric flask was shaken for 1 min using vortex mixer. The volume was made up to $10 \mathrm{ml}$. Then the solution was filtered and diluted and the concentration of GLZ was determined spectrophotometrically at $229 \mathrm{~nm}$ [9].

$$
\text { Loading efficiency }=\frac{\text { Actual drug content in nanosponge }}{\text { Theoretical drug content }} \times 100
$$

\section{Determination of drug content}

To study the amount of drug incorporated in the nanosponges, GLZ was extracted from the nanosponges by dissolving them in $25 \mathrm{ml}$ Methanol. The resulting solution was filtered through a 0.45 -micron membrane filter. The GLZ content in the methanolic extracts was analyzed spectrophotometrically by using a UV-Visible spectrophotometer (UV Jasco V630) at a wavelength of $229 \mathrm{~nm}$, against methanol as blank [10].

$$
\% \text { drug content }=\left(\frac{\mathrm{Wa}}{\mathrm{Wt}}\right) \times 100
$$

Where,

$$
\begin{aligned}
& \mathrm{Wa}=\text { Actual drug content and } \\
& \mathrm{Wt}=\text { Theoretical drug content }
\end{aligned}
$$

\section{Determination of particle size}

The size of particles is maintained during polymerization for the formation of free-following powders having fine aesthetic attributes. Particle size analysis of loaded and unloaded nanosponges performed by laser light diffractometry or Malvern zeta sizer. The cumulative graph is maintained or plotted as particle size against time to study effect of particle size on drug release [11].

\section{In vitro drug release studies}

In vitro drug release studies were performed in triplicate using USP Paddle method at $50 \mathrm{rpm}$ and $37 \pm 0.2^{\circ} \mathrm{C}$ in $900 \mathrm{ml}$ of phosphate buffer (pH 6.8) [12]. $100 \mathrm{mg}$ of the formulated nanosponges is used for each experiment. Samples were taken at appropriate time intervals for $1 \mathrm{~h}$ interval for $10 \mathrm{hr}$. The samples were measured spectrophotometrically at $229 \mathrm{~nm}$. Fresh dissolution medium was replenished each time when sample is withdrawn to compensate the volume.

\section{Fourier-transform infrared analysis}

Fourier-transform infrared (FTIR) spectra of pure GLZ and pure polymer (Eudragit), and nanosponges of drug GLZ was taken to access interaction if any interaction between drug and polymer in mixtures. The scanned mixture by using an FTIR (Jasco- 460 plus). The FTIR spectra of mixtures were compared with that of the pure drug and Polymer to assess any change in the principle peaks of spectra of pure drug and polymer [13].

\section{Differential scanning calorimetry}

Differential scanning calorimetric (DSC) studies of pure GLZ, pure polymer (Eudragit S100) and nanosponges of drug GLZ with Eudragit S100 was performed to assess what changes happen during the heat change and thermal behavior of the drug is also determine. The pure drug sample shows sharp endothermic peak at melting point of drug. The samples were kept on DSC reference pan and DSC curves were obtained by differential scanning calorimeter (DSC 60; Shimadzu) at a heating rate of $10^{\circ} \mathrm{C} / \mathrm{min}$ from 0 to $300^{\circ} \mathrm{C}$ in nitrogen atmosphere [14].

\section{Scanning electron microscopy studies}

Scanning electron microscopy (JSM-5200, Tokyo Japan) was used to analyze particle size and surface topography was operated at $15 \mathrm{kV}$ acceleration voltage. A concentrated aqueous suspension was spread over a slab and dried under vacuum. The sample was shadowed in a cathodic evaporator with a gold layer $20 \mathrm{~nm}$ thick. Photographs were elaborated by an image processing program and individual NP diameters were measured to obtain mean particle size.

Nanosponges that showed the best results in the solubility and dissolution studies were subjected to scanning electron microscopy (SEM) studies to confirm the changes mounting made during the formation of nanosponges. Samples were prepared by powder onto a brass stub using graphite glue and coated with gold under vacuum before use. Images were recorded at the required magnification at an acceleration voltage of $10 \mathrm{KV}$ using a scanning electron microscope [15]. 


\section{Stability studies}

Accelerated stability studies: $-40{ }^{\circ} \mathrm{C} \pm 2{ }^{\circ} \mathrm{C} / 75 \% \mathrm{RH} \pm 5 \% \mathrm{RH}$. As per ICH guidelines, the samples for stability analysis must be exposed to an environment of $4 \% \pm 2^{\circ} \mathrm{C} / 75 \% \mathrm{RH} \pm 5 \% \mathrm{RH}$ for a period of 6 mo. As per the standard protocol, the samples must be analysed at $0,1,2,3$-and 6-months' time points. Accelerated stability studies were performed for the final optimized formulation. Samples were analysed at 1,2 mo' time points [16].

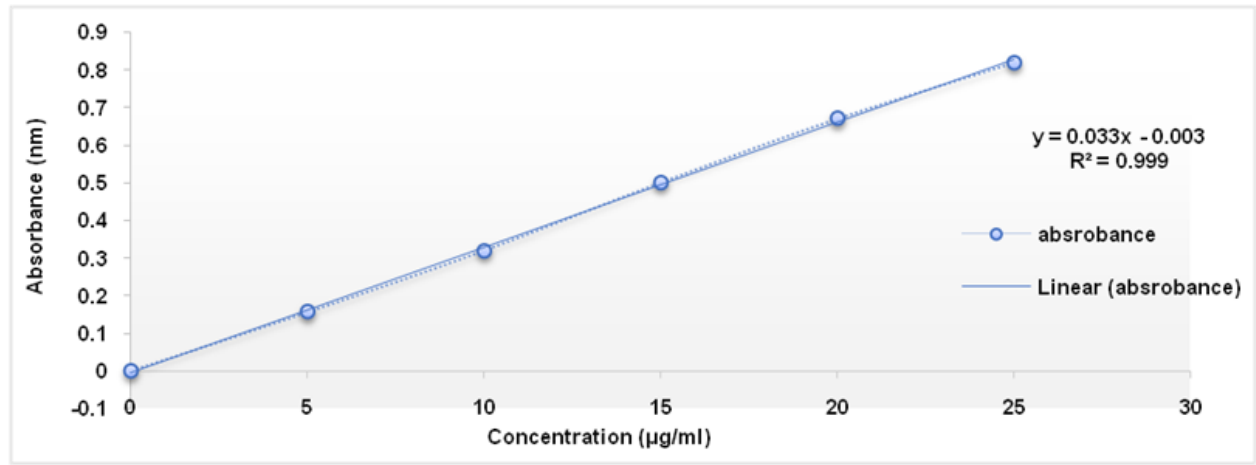

Fig. 1: Calibration curve of GLZ by UV method

\section{RESULTS}

\section{Preformulation studies}

Calibration curve of GLZ

\section{Evaluation of nanosonges}

\section{Production yield}

The production yield (PY) can be determined by calculating the initial weight of raw materials and final weight of nanosponges.
The percentage yield of different batches was determined by weighing the nanosponges after drying. The percentage yields of different formulations were found to be in the range of $73.8 \pm 0.30 \%$ $85.6 \pm 0.32 \%$ as shown in below table 2 .

\section{Drug entrapment efficiency}

The drug entrapment efficiency found in the range of $62.8 \pm 0.22$ to $73.1 \pm 0.39$. The increases concentration of polymer the drug entrapment efficiency also increases.

Table 2: Percentage yield of different batches of nanosponges

\begin{tabular}{lll}
\hline S. No. & Batch & Yield (\%) \\
\hline 1 & F1 & $73.8 \pm 0.30$ \\
2 & F2 & $75.2 \pm 0.25$ \\
3 & F3 & $85.6 \pm 0.32$ \\
4 & F4 & $78.9 \pm 0.18$ \\
5 & F5 & $80.1 \pm 0.16$ \\
\hline
\end{tabular}

Average of three values $\pm S D n=3$

Table 3: Drug entrapment efficiency of different batches of nanosponges

\begin{tabular}{lll}
\hline S. No. & Batch & Entrapment efficiency (\%) \\
\hline 1 & F1 & $62.8 \pm 0.22$ \\
2 & F2 & $66.2 . \pm 0.25$ \\
3 & F3 & $70.6 \pm 0.77$ \\
4 & F4 & $71.8 \pm 0.36$ \\
5 & F5 & $73.1 \pm 0.39$ \\
\hline
\end{tabular}

Average of three values \pm SD $n=3$

\section{Determination of drug content}

The various batches of the nanosponges were subjected for drug content analysis. The powdered nanosponges $(30 \mathrm{mg})$ were dissolved in adequate quantity $(100 \mathrm{ml})$ of phosphate buffer PH 6.8 then filter. The UV absorbance of the filtrate was measured using a UV spectrophotometer at $229 \mathrm{~nm}$.

The drug content of different formulation was found to be in the range of $77.21 \pm 0.17$ to $90.62 \pm 0.25$ as shown in below table 4 .

Table 4: Drug content values of different batches of nanosponges

\begin{tabular}{lll}
\hline S. No. & Batch & Drug content (\%) \\
\hline 1 & F1 & $77.21 \pm 0.17$ \\
2 & F2 & $83.36 \pm 0.11$ \\
3 & F3 & $90.62 \pm 0.25$ \\
4 & F4 & $85.32 \pm 0.13$ \\
5 & F5 & $86.92 \pm 0.09$ \\
\hline
\end{tabular}

Average of three values \pm SD $n=3$ 


\section{Particle size analysis}

The average particle size was obtained in range of $303 \pm 2.36 \mathrm{~nm}$ to $680 \pm 2.50 \mathrm{~nm}$. The change in the concentration of polymer results in variation of particle size of nanosponges.
The average particle size of formulation batch F3 showed minimum particle size i.e. $418 \pm 2.23 \mathrm{~nm}$ while formulation batch F5 showed maximum particle size i.e. $680 \pm 2.50 \mathrm{~nm}$. An increase in the concentration of polymer leads to increase in the particle size of nanosponges.

Table 5: Particle size of different batches of nanosponges

\begin{tabular}{lll}
\hline S. No. & Formulation code & Particle size (nm) \\
\hline 1 & F1 & $303 \pm 2.36$ \\
2 & F2 & $380 \pm 2.59$ \\
3 & F3 & $418 \pm 2.23$ \\
4 & F4 & $519 \pm 2.66$ \\
5 & F5 & $680 \pm 2.50$ \\
\hline
\end{tabular}

Average of three values \pm SD $n=3$

\section{In vitro drug release study}

In vitro drug release for GLZ loaded nanosponges for a period of 10 h, was carried out by using Phosphate buffer ( $\mathrm{pH}$ 6.8) at $37 \pm 50 \circ \mathrm{C}$ using cellophane membrane of $0.22 \mathrm{~nm}$. From the dissolution profile of formulations F1 to F5, it is concluded that formulation batch F3 shows a better drug release profile than other formulations. Cumulative \% release has been shown for average of three preparations, Cumulative \% drug release for all the formulations are depicted in the table 6 .

Table 6: In vitro drug release studies

\begin{tabular}{|c|c|c|c|c|c|}
\hline \multirow[t]{2}{*}{ Time in (h) } & \multicolumn{5}{|c|}{ \% Drug release of nanosponges } \\
\hline & $1: 1$ & $1: 2$ & $1: 3$ & $1: 4$ & $1: 5$ \\
\hline 1 & $10.07 \pm 0.90$ & $11.99 \pm 1.12$ & $15.25 \pm 0.78$ & $13.36 \pm 1.42$ & $10.04 \pm 0.92$ \\
\hline 2 & $15.32 \pm 0.85$ & $23.58 \pm 0.80$ & $19.48 \pm 0.82$ & $20.22 \pm 1.36$ & $20.53 \pm 1.00$ \\
\hline 3 & $25.36 \pm 0.92$ & $32.72 \pm 0.99$ & $33.79 \pm 1.02$ & $31.98 \pm 0.96$ & $31.76 \pm 1.14$ \\
\hline 4 & $39.98 \pm 0.65$ & $40.347 \pm 1.2$ & $51.93 \pm 0.98$ & $42.46 \pm 1.22$ & $40.29 \pm 1.50$ \\
\hline 5 & $49.92 \pm 1.12$ & $50.38 \pm 1.50$ & $65.03 \pm 1.14$ & $56.34 \pm 1.33$ & $44.42 \pm 1.04$ \\
\hline 6 & $55.23 \pm 1.01$ & $58.3 \pm 0.98$ & $69.3 \pm 1.32$ & $65.72 \pm 1.18$ & $50.97 \pm 1.22$ \\
\hline 7 & $63.74 \pm 0.58$ & $64.71 \pm 1.11$ & $77.74 \pm 1.26$ & $71.12 \pm 1.08$ & $61.36 \pm 1.16$ \\
\hline 8 & $70.98 \pm 1.20$ & $75.98 \pm 0.96$ & $82.15 \pm 1.08$ & $77.92 \pm 1.12$ & $76.32 \pm 1.32$ \\
\hline 9 & $75.26 \pm 0.74$ & $81.52 \pm 1.25$ & $85.78 \pm 0.98$ & $86.24 \pm 0.90$ & $84.28 \pm 1.24$ \\
\hline 10 & $84.32 \pm 0.62$ & $87.36 \pm 1.32$ & $94.40 \pm 1.12$ & $91.40 \pm 0.88$ & $90.32 \pm 1.20$ \\
\hline
\end{tabular}

Average of three values $\pm S D n=3$

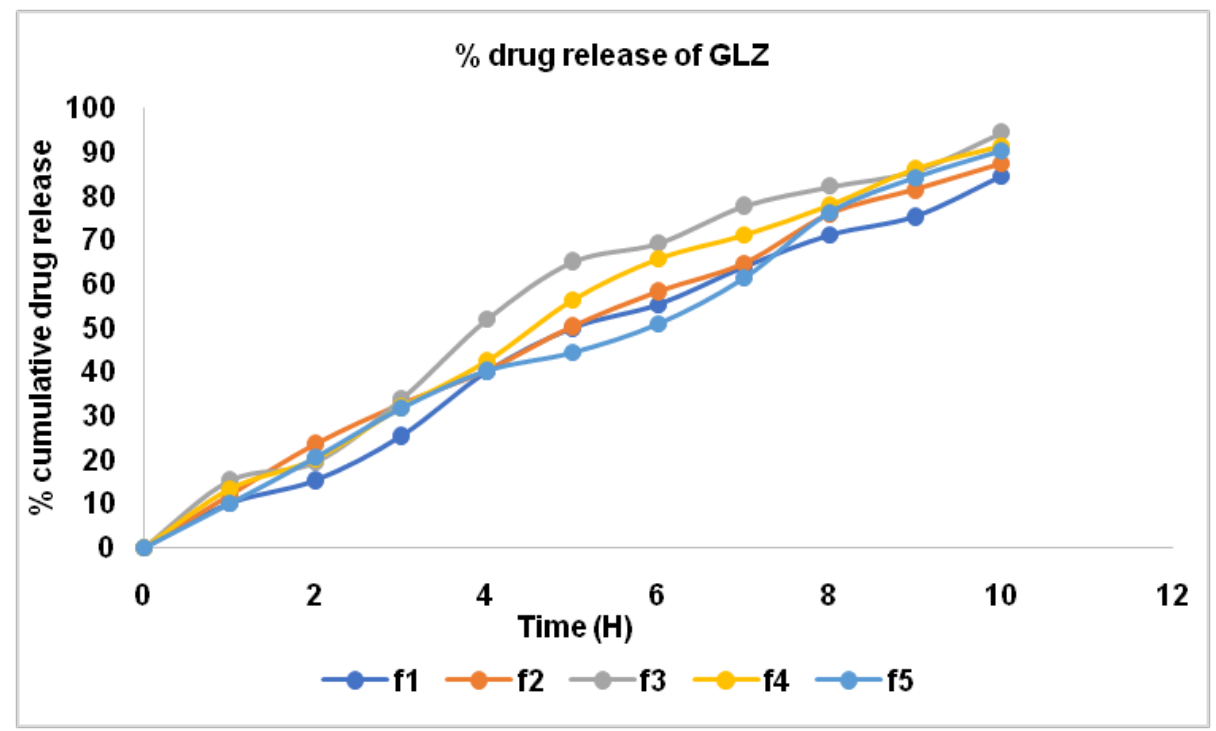

Fig. 2: Graphical presentation of $\%$ drug release of F1 to F5 batches

\section{Modeling of dissolution profile}

In the present study, different release kinetic equations (zero order, first order, Higuchi equation and Korsmeyer-peppas equation) were applied to interpret the release rate of drug from nanosponges. The data of in vitro release were fitted to these models and equations to explain the release kinetics of GLZ from nanosponges [17]. 


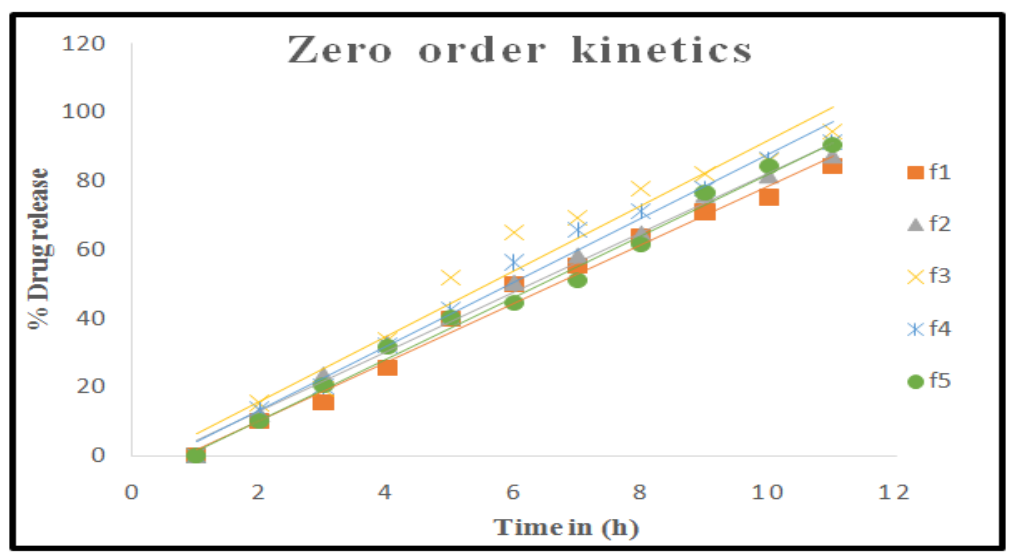

Fig. 3: Zero order release kinetics

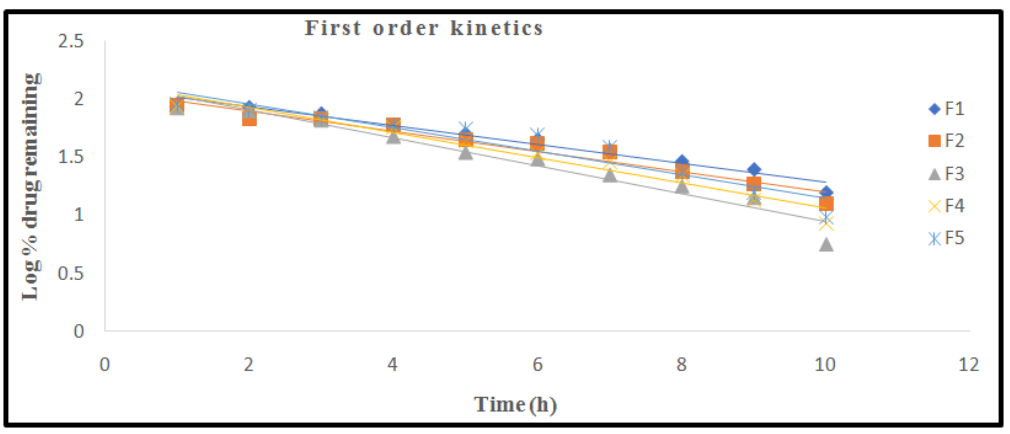

Fig. 4: First order release kinetics

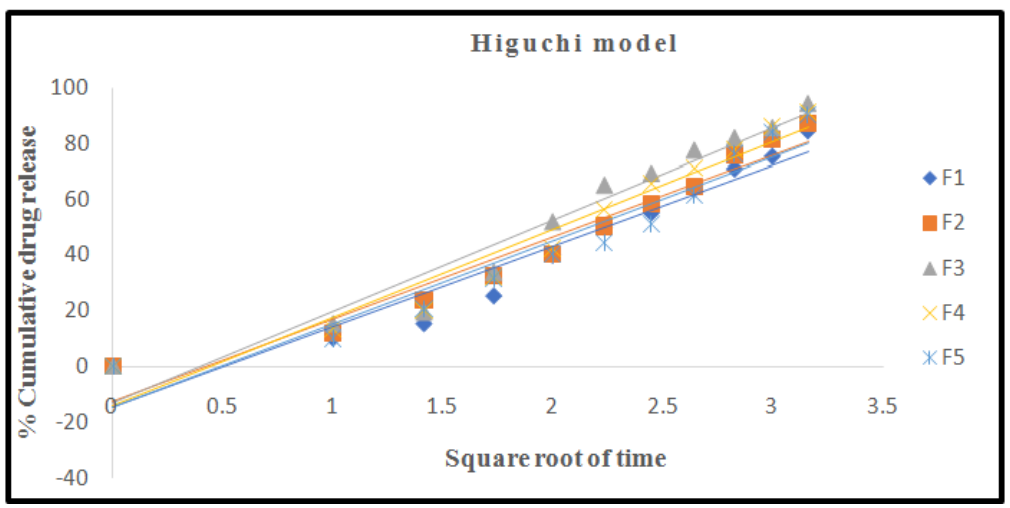

Fig. 5: Higuchi model

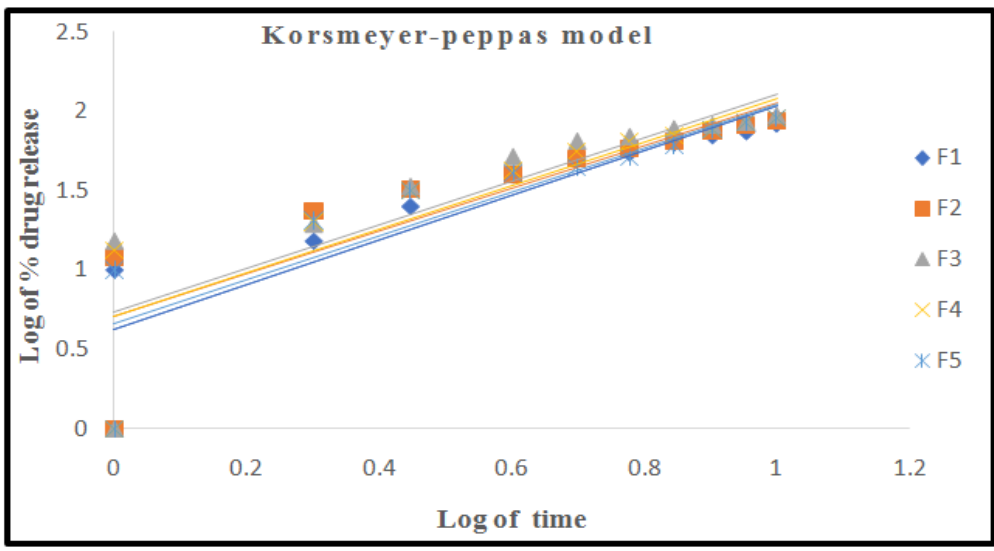

Fig. 6: Korsmeyer-peppas model 
Table 7: $\mathbf{R}^{2}$ values of different release kinetic models

\begin{tabular}{lllll}
\hline Formulation & Zero order kinetics & First order kinetics & Higuchi model & Korsmeyer-Peppas model \\
\cline { 2 - 5 } & $\mathbf{R}^{\mathbf{2}}$ & $\mathbf{R}^{\mathbf{2}}$ & $\mathbf{R}^{\mathbf{2}}$ & \\
\hline F1 & 0.9823 & 0.9529 & 0.9326 & 0.8079 \\
F2 & 0.9612 & 0.9462 & 0.9524 & 0.7541 \\
F3 & 0.9927 & 0.9711 & 0.9475 & 0.7425 \\
F4 & 0.9865 & 0.9576 & 0.9462 & 0.7608 \\
F5 & 0.9923 & 0.9021 & 0.9205 & 0.7878 \\
\hline
\end{tabular}

The in vitro release profile of the formulations indicates that the rate of drug release was higher for formulations, among all the formulations F3 shows more amount of drug release with the medium amount of polymer ratio. The plot of time vs cumulative $\%$ drug release in the zero-order kinetic model the regression coefficient value shows linearity as shown in (fig 3 ). The slopes and regression coefficient values $\left(\mathrm{R}^{2}\right)$ of various mathematical models for optimized batch F3, 0.9927, 0.9711, 0.9475, 0.7425 for zero order, first order, Higuchi and Peppas model respectively. The good linearity observed with the zero-order and regression coefficient value is higher. Hence this formulation is best fitted into zero-order release model.

\section{FT-IR studies}

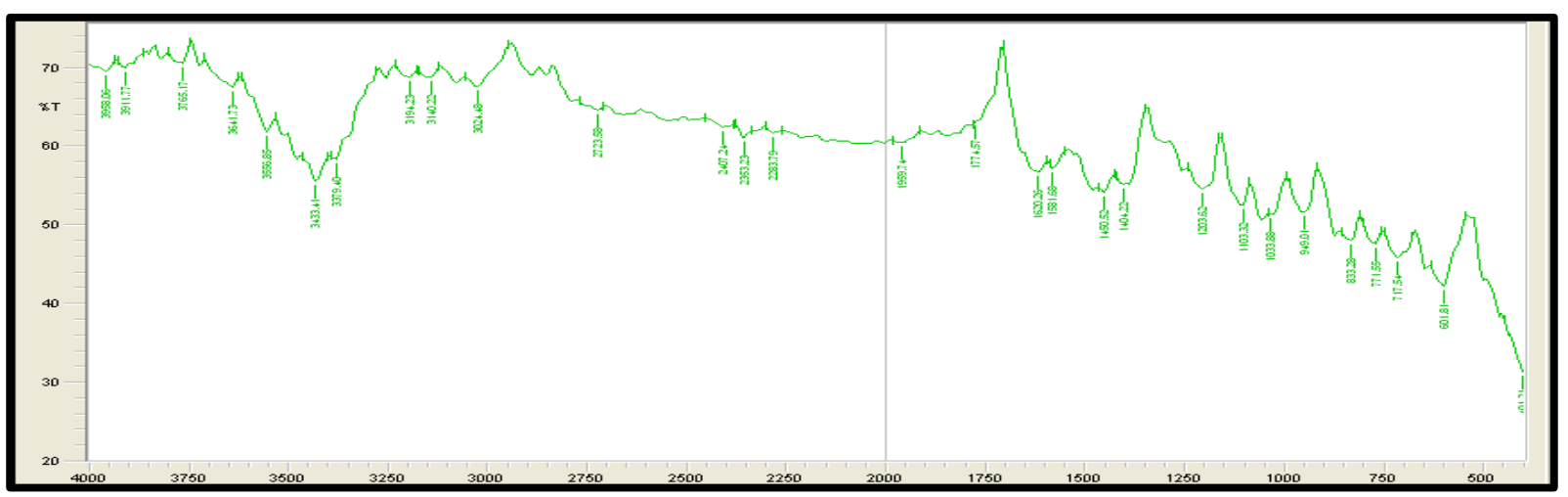

Fig. 7: FT-IR spectra of pure GLZ

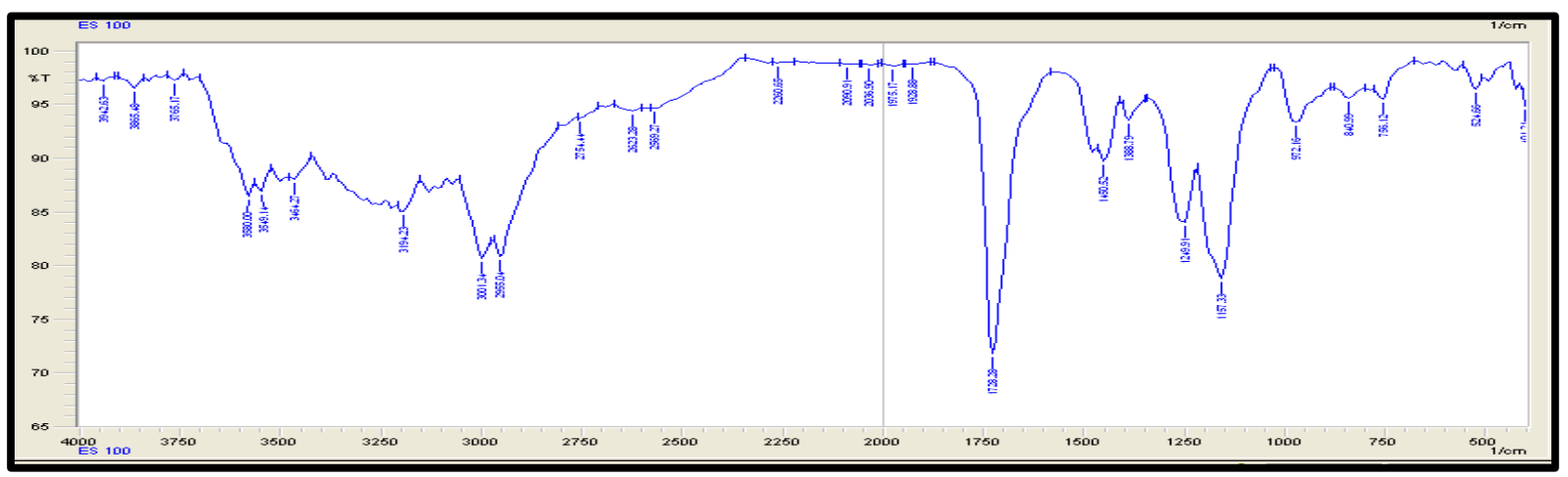

Fig. 8: FT-IR spectra of Eudragit S100

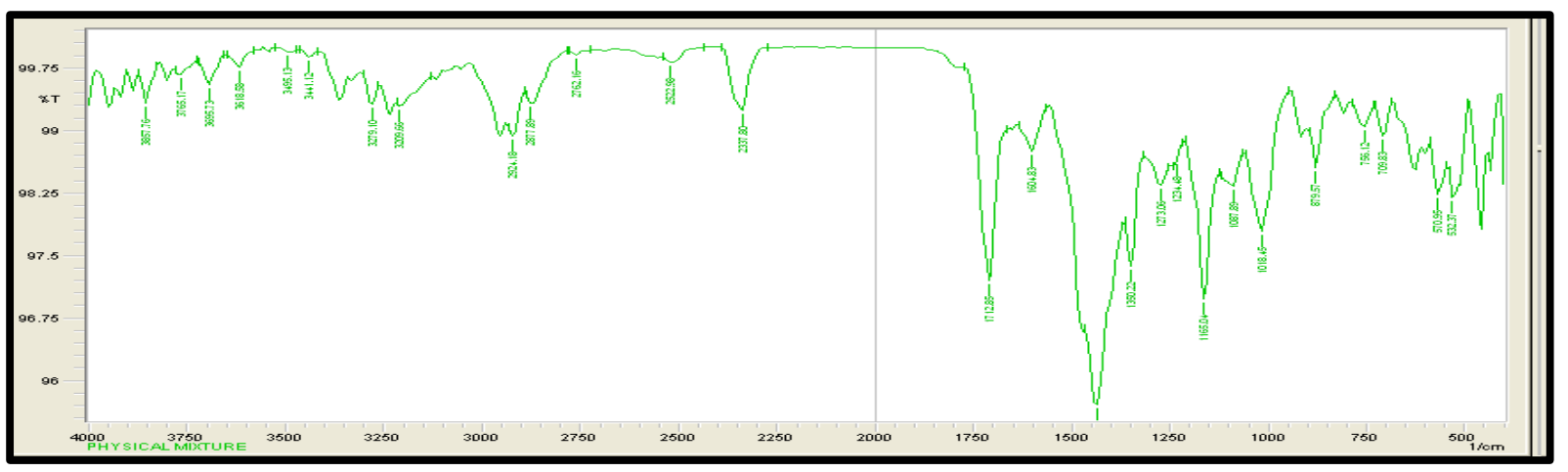

Fig. 9: FT-IR spectra of the physical mixture (drug+polymer) 


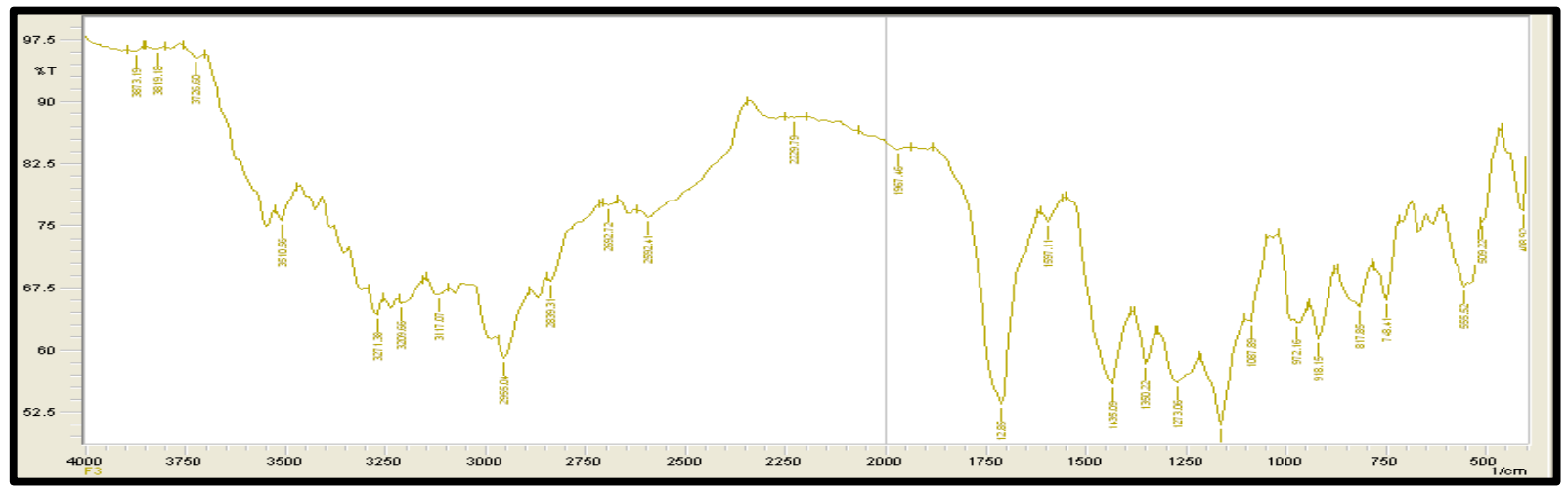

Fig. 10: FT-IR spectra of prepared nanosponges

DSC studies

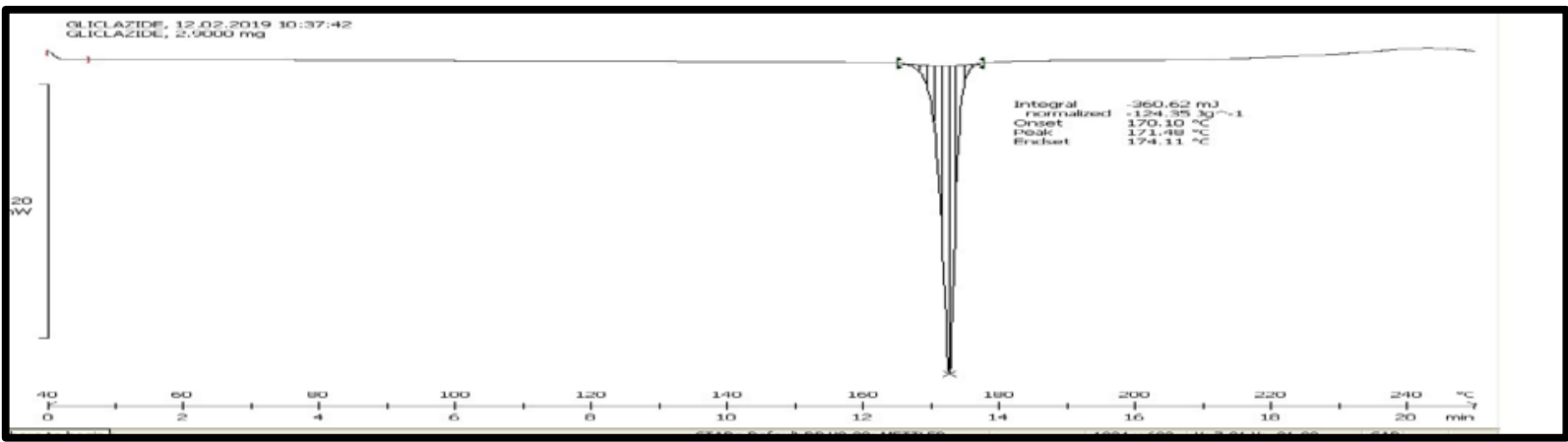

Fig. 11: DSC thermogram of Pure GLZ

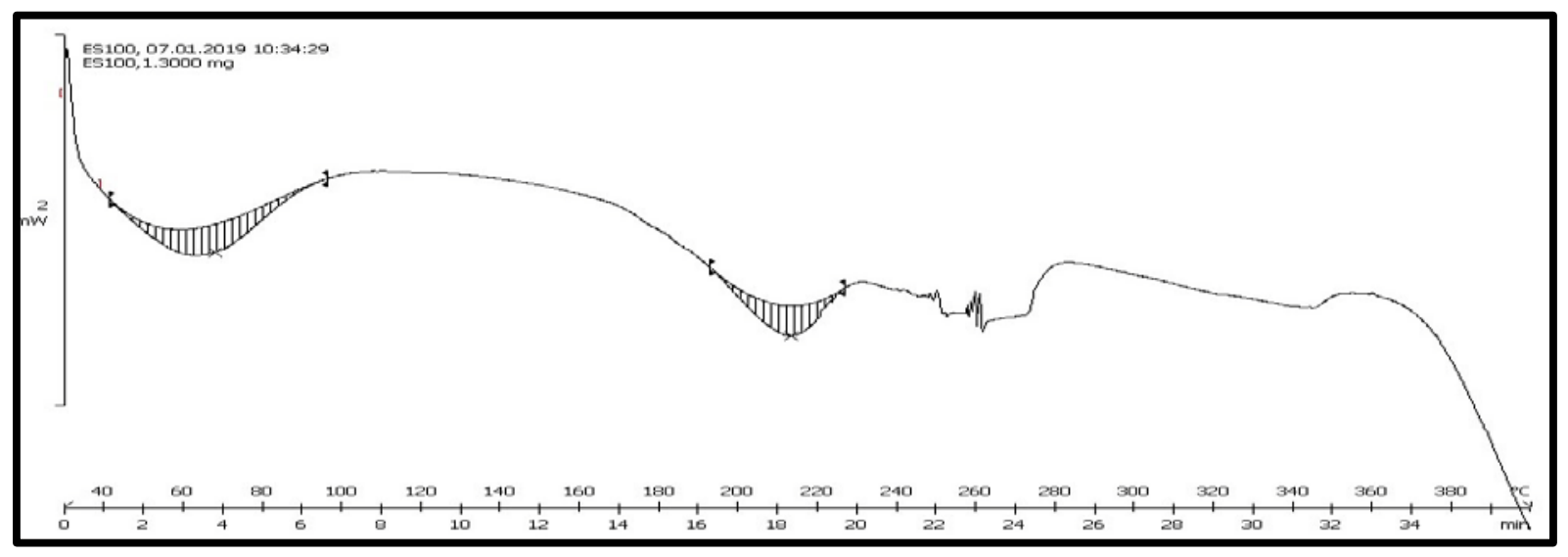

Fig. 12: DSC thermogram of polymer

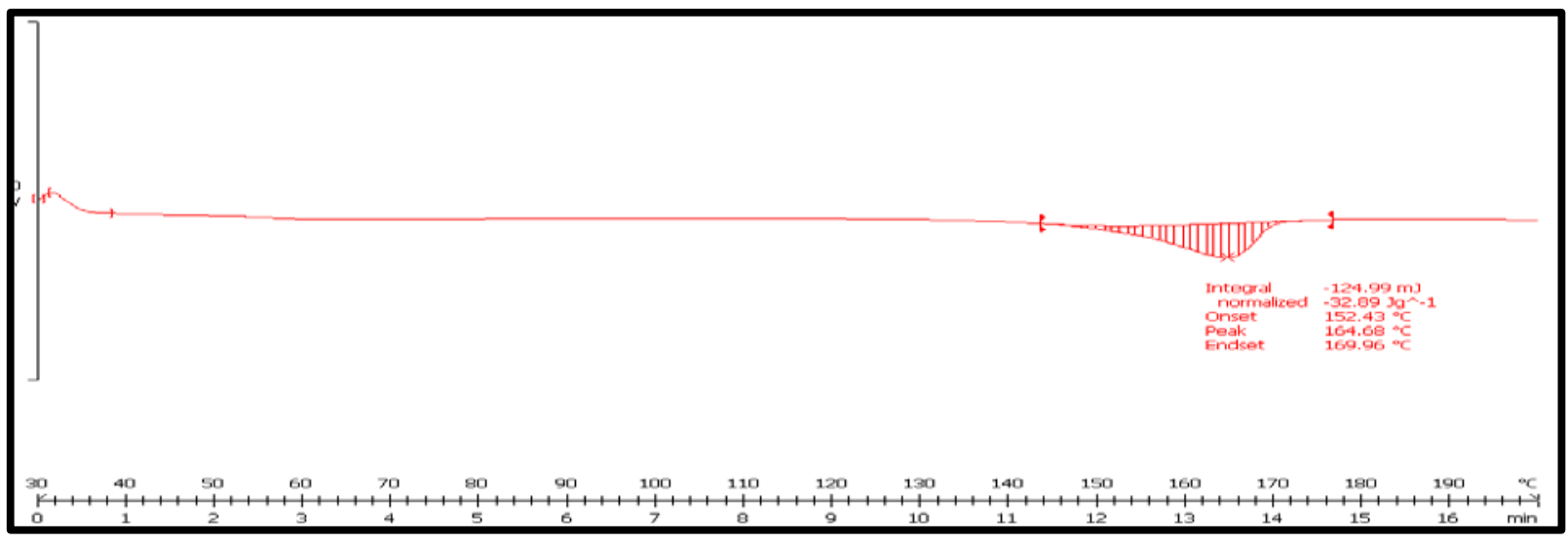

Fig. 13: DSC thermogram of the physical mixture (drug+polymer) 


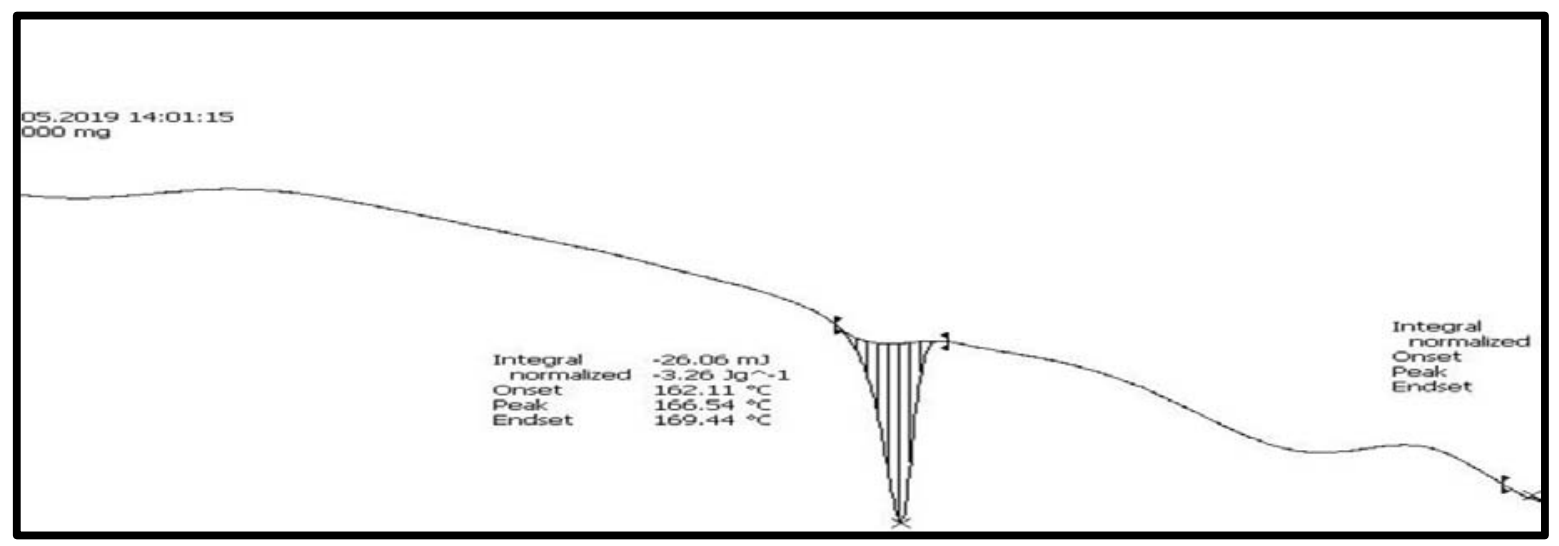

Fig. 14: DSC thermogram of prepared nanosponges

\section{SEM studies}

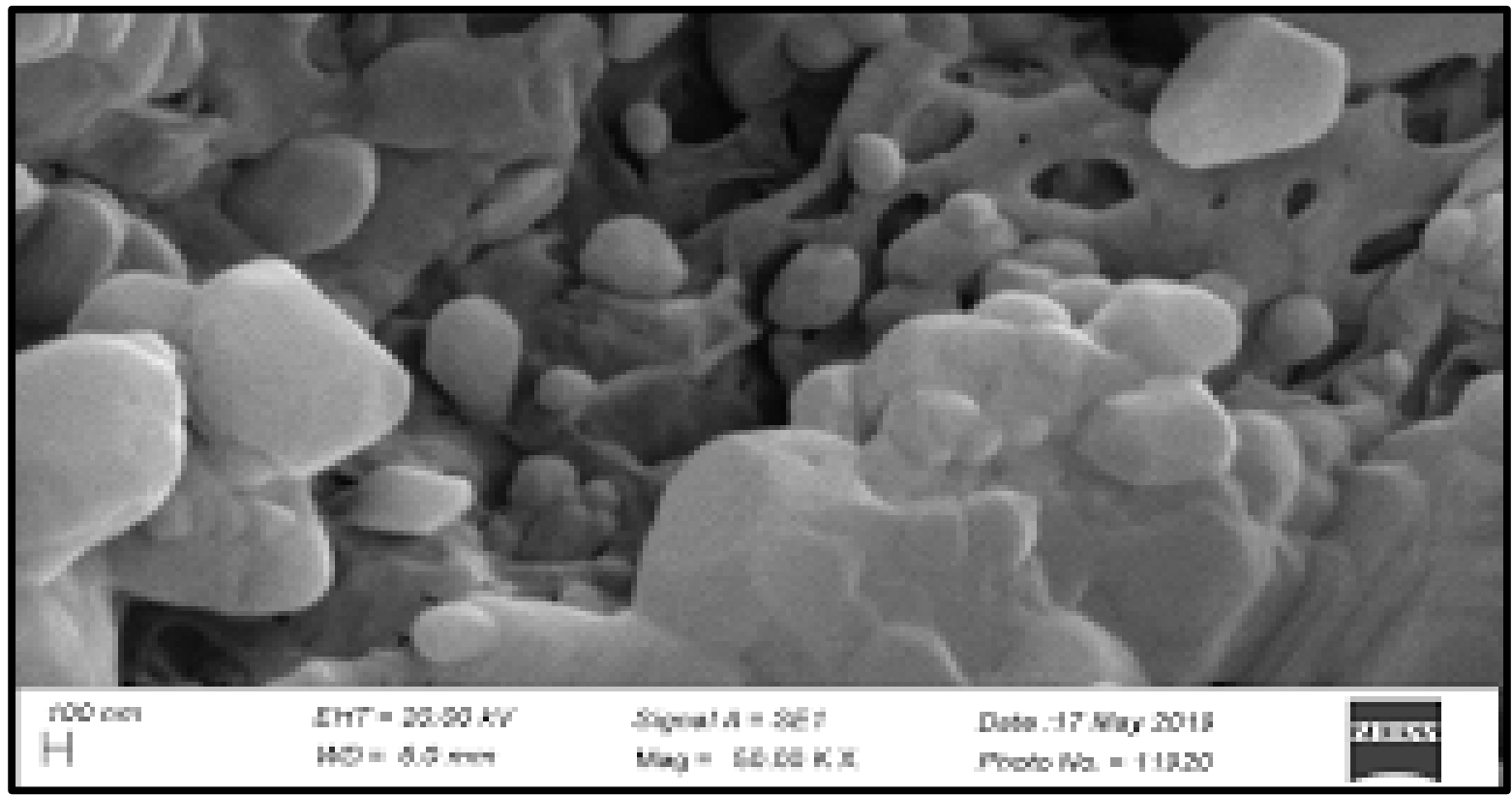

Fig. 15: SEM image of nanosponges

\section{Stability studies}

Optimized formulation was subjected to stability studies as per ICH guidelines. Various parameters such as drug content, and in vitro drug release were measured before and after 30,60, days of stability. Results of stability studies are shown in below table 8 .
Results of stability studies showed there is no significant change in the above mentioned parameter after elevated temperature and humidity conditions during stability studies. Thus, it can be proved from the stability studies that the prepared formulation is stable and not much affected by elevated humidity and temperature conditions Stability studies.

Table 8: Stability study of optimized formulation

\begin{tabular}{lll}
\hline Time (day) & Drug content (\%) & In vitro drug release (\%) \\
\hline 0 & $98.86 \pm 0.68$ & $99.85 \%$ \\
30 & $98.20 \pm 0.99$ & $99.74 \%$ \\
60 & $98.30 \pm 1.50$ & $99.51 \%$ \\
\hline
\end{tabular}

\section{DISCUSSION}

The calibration curve of GLZ by UV method showed the linearity in absorbance and coefficient of regression found to be 0.9994 . The IR studies shows that there is no interaction found between drug and excipients. All the characteristic peaks of GLZ were present in the spectrum of drug (fig. 7) and prepared GLZ nanosponges (fig. 10), indicating compatibility in drug and polymer the spectrum confirmed that there is no significant change in the chemical integrity of the drug [18]. The DSC thermogram pure GLZ shows sharp endothermic peak at $17^{\circ} \mathrm{C}$, which is its melting point as it melts with decomposition [19]. Such a sharp endothermic peak indicates that GLZ used was in the pure crystalline state. The thermogram of prepared GLZ nanosponges 1:3 (fig. 14) displayed the nanosponge structure is amorphous as no sharp melting peak is shown. 
The percentage yield of different batches was determined by weighing the nanosponges after drying. The percentage yields of different formulations were found to be in the range of $73.8 \pm 0.30 \%$ $85.6 \pm 0.32 \%$. The drug entrapment efficiency found in the range of $62.8 \pm 0.22$ to $73.1 \pm 0.39$. The increases concentration of polymer the drug entrapment efficiency also increases. The drug content of different formulation was found to be in range of $77.21 \pm 0.17$ to $90.62 \pm 0.25$. The average Particle size was obtained in range of $303 \pm 2.36 \mathrm{~nm}$ to $680 \pm 2.50 \mathrm{~nm}$. The Change in the concentration of polymer results variation in particle size of nanosponges [20]. The average particle size of formulation batch $\mathrm{F} 1$ showed minimum particle size i.e. $303 \pm 2.36 \mathrm{~nm}$ while formulation batch $\mathrm{F} 5$ showed maximum particle size i.e. $680 \pm 2.50 \mathrm{~nm}$. An increase in the concentration of polymer leads to increase in the particle size of nanosponges. The SEM of the prepared nanosponges revealed a porous nature of GLZ nanosponges (fig. 15). Drug release data for the nanosponge formulation fitted into various release kinetic equations to find out order of drug release. The various release kinetic curves are shown in fig. 3-7. The correlation coefficient showed that the release profile followed the zero-order drug release $\left(R^{2}=0.9927\right)$ from these results it is apparent that the regression coefficient value closer to unity in case of zero order kinetic model. The data indicate more linearity when plotted by the zero-order kinetic model. Hence, it can be concluded that the major mechanism of drug release follows zero-order kinetics [21].

\section{CONCLUSION}

Nanosponge is the best novel drug delivery system. For improving the dissolution and bioavailability profile of poorly water-soluble drug. BCS class-2 drug are more suitable drug candidate for formulated into nanosponges. Nanosponges are able to encapsulate variety of various types of drug molecules. Nanosponges prepared by using emulsion solvent diffusion method are simpler and production cost of method is less. Nanosponge prepared by emulsion solvent diffusion method shows good entrapment efficiency ranged between $62.8 \pm 0.22$ to $73.1 \pm 0.39$ and optimum drug release is $94.40 \%$. The compatibility of drug and polymer determine by FT-IR, and DSC study. The result of IR and DSC shows drug and polymer are compatible to each other. The nanosponge formulation shows zero order drug release. The outcome of the study concluded that Eudragit S100 are employed as polymer for oral drug delivery system. Nanosponges of gliclazide increases solubility and dissolution rate of drug. The emulsion solvent diffusion method is best method for preparation of nanosponges.

\section{AUTHORS CONTRIBUTIONS}

All the author have contributed equally

\section{CONFLICT OF INTERESTS}

\section{Declared none}

\section{REFERENCES}

1. Bhowmik H, Venkatesh ND, Kuila A, Kumar HK. Nanosponge: a review. Int J Appl Pharm 2018;10:1-5.

2. Subramanian S, Singireddy S, Krishnamoorthy K, Rajappan M. Nanosponges: a novel class of drug delivery system review. J Pharm Sci 2012;15:103-11.
3. Cavalli R, Trotta F, Tumiatti W. Cyclodextrin-based nanosponges drug delivery. J Inclusion Phenom Macrocyclic Chem 2006;56:209-13.

4. Sharma R, Roderick B Walker, Kamla P. Evaluation of kinetics and mechanism of drug release from econazole nitrate nanosponge loaded carbopol hydrogel. Ind J Pharm Edu Res 2011;45:25-31.

5. Kilicarslan M, Baykara T. The effect of drug-polymer ratio on the properties of verapamil loaded microspheres. Int J Pharm 2003;252:99-109.

6. Pentewar RS, Kaji S, Bharati R, MDS technology: an approach for topical, oral controlled and cosmetic formulations. Res J Pharm Biol Chem Sci 2014;5:1170.

7. Sehgal N, Gupta V, Kanna S. A review on nanosponges: a boon to targeted drug delivery for an anticancer drug. Asian J Pharm Clin Res 2019;12:1-7.

8. Salunkhe A, Kadam S, Magar S, Dangare K. Nanosponges: a modern formulation approach in drug delivery system. World J Pharm Pharm Sci 2018;7:575-92.

9. Swetha A, Gopal Rao M, Venkata Ramana K, Niyaz Basha B, Koti Reddy V. Formulation and in vitro evaluation of etodolac entrapped in microsponge based drug delivery system. Int J Pharma 2011;1:73-90.

10. Swaminathan S, Vavia PR, Trotta F, Torne S. Formulation of Bcyclodextrin based nanosponges of itraconazole. J Incl Phenom Macrocycl Chem 2007;57:89-94.

11. Arvapally S, Harini M, Harshitha G, Arun Kumar A. Formulation and in vitro evaluation of glipizide nanosponges. Am J Pharmtech Res 2017;7:341-61.

12. Kumar AN. Enhancement of the dissolution rate of glibenclamide using liquisolid technique. Int J Res Pharm Nano Sci 2015;4:206-16.

13. Singh R, Bharati N, Madan J, Hiremath SN. Characterization of cyclodextrin inclusion complexes-a review. J Pharm Sci Tech 2010;2:171-83

14. Swaminathan S, Pastero L, Serpe L, Trotta F, Vavia P, Aquilano $\mathrm{D}$, et al. cyclodextrin-based nanosponges encapsulating camptothecin: physicochemical characterization, stability and cytotoxicity. Eur J Pharm Biopharma 2010;74:193-201.

15. Dubey P, Sharma HK, Shah S, Tyagi CK, Chandekar A. Jadon SR. Formulation and evaluation of cyclodextrin complexed ceadroxil loaded nanosponges. Int J Drug Delivery 2017;9:84-100.

16. ICH, Q1A, (R2) Stability testing guidelines: stability of new drug substances and product: methodology in processing of ICH Geneva; 2003.

17. Paulo Costa, Jose Manuel Sousa Lobo. Modelling and comparison of dissolution profiles. Eur J Pharm Sci 2001;13:123-33.

18. Varma Mohan M, Kumar Satish P. Formulation and evaluation of gliclazide tablet containing PVP-K30 hydroxypropyl- $\beta$ cyclodextrin solid dispersion. Int J Pharm Sci Nanotechnol 2012;5:1706-19.

19. Biswal S, Sahoo J, Murthy PN. Characterization of gliclazide-PEG 8000 solid dispersions. Tropical J Pharm Res 2009;8:417-24.

20. Keerthi Priya Darshaini M, Jyothshna Devi K, Shilpaja C, Usmankar K. Atorvastatin loaded nanosponges-a novel strategic approach for enhanced bioavailability. World J Pharm Pharm Sci 2017;6:1223-36.

21. Asad M, Bashir S, Mahmood T, Nazir I, Imran M, karim S, et al. Fabrication and characterization of gliclazide loaded microcapsules. Braz Arch Biol Technol 2014;57:874-81. 